\title{
LOS INICIOS DEL LITTLE THEATRE MOVEMENT EN FRANCIA
}

CRISTINA NAVAS ROMERO 1

Universidad de Málaga

A finales del siglo XIX, apareció un movimiento teatral experimental que transformó el teatro moderno y reavivó la dramaturgia en Europa y en los Estados Unidos. Este movimiento, llamado Free Theatre o Independent Theatre en Europa y Little Theatre en los Estados Unidos, se originó en los teatros no comerciales y sus miembros eran artistas, mayormente inmigrantes y aficionados, que rechazaban los convencionalismos y los prejuicios teatrales de épocas anteriores.

Este movimiento surgió en oposición al teatro comercial, centrado principalmente en la obtención de grandes beneficios en las ventas de taquilla. En el teatro experimental se pretendía la creación de un teatro más creativo y estaba formado por un grupo de artistas, cuyo nexo de unión era la pasión por el teatro y para quiénes los beneficios en la taquilla no eran una prioridad. En cuanto a las características de estos teatros anti-comerciales, destacaba el tamaño, pues eran de dimensiones bastante pequeñas, dado que sus fundadores habían pensado en la creación de un escenario más íntimo, en el que existiese una cercanía mayor entre los actores y el público, que la que poseían los teatros comerciales. Sin embargo, el tamaño de sus teatros variaba considerablemente: existían locales que poseían un aforo de 70 butacas, mientras que en otros era de 300. Otros dos aspectos que distinguían a estos teatros eran, en primer lugar, el repertorio, que estaba formado por una gran variedad de obras, con una cartelera que se cambiaba dos o tres veces

\footnotetext{
1 Profesora de la Universidad de Málaga. Correo-e: cnavas@uma.es. Recibido: 06-02-2009; segunda versión: 14-04-2009.
} 
por semana, por lo que se ofrecía mayor variedad dramática que en los teatros comerciales y, en segundo lugar, la experimentación, que constituía una de las peculiaridades más notables de sus obras. Además, en estos teatros no se vendían las entradas en la taquilla, sino que se financiaban mediante la subscripción de sus socios, por lo tanto, evitaban la censura de las obras, al no desarrollar una actividad comercial.

El movimiento teatral del Little Theatre apareció en los Estados Unidos en 1912; sin embargo, no tuvo su origen en el continente americano, sino que surgió en Europa en 1887, para implantarse posteriormente en Norteamérica. A pesar de que el primer teatro experimental se fundó en Francia, el origen de este movimiento no se situaba en el país galo, sino que procedía de los países escandinavos, donde surgieron tres autores decisivos en el nacimiento de la dramaturgia moderna y en la aparición de este movimiento teatral anti-comercial. El primero de estos dramaturgos, así como el más importante, fue Henrik Ibsen, descubridor de una nueva forma de expresión teatral:

the new conception to which he [Ibsen] turned instead was indeed not slow to conquer the resistance of Scandinavia, Europe, eventually the whole world. It has revolutionized the art of the actor as well as of the dramatist. Far more than this, it has been one of the prime levers of the social revolution which is sweeping over us². (Heller, 1921: 87).

El traductor y crítico William Archer, gran estudioso de Ibsen, consideraba al dramaturgo noruego precursor del movimiento teatral del Little Theatre y destacaba a su obra Ghosts (1881) con respecto al resto de su producción dramática. Es importante señalar que Ghosts fue una de las pocas obras de teatro que produjeron el día de su estreno tres de las primeras compañías de Little Theatre aparecidas en Europa. Ibsen debió de influir sobremanera en el pensamiento de estos artistas. Los fundadores de estas compañías conocían perfectamente la obra dramática de Ibsen, mucho antes de

2 Traducción de la cita: "la nueva concepción que él (Ibsen) fomentaba conquistó rápidamente el rechazo de Escandinavia, Europa y, finalmente, del resto del mundo. Esta concepción ha revolucionado la maestría del actor y del dramaturgo. No sólo eso, sino que ésta ha sido una de las principales impulsoras de la revolución social que nos está arrasando". 
que se establecieran las primeras compañías, pues existía una producción impresa de su obra literaria y un gran número de traducciones. Huelga decir que este dramaturgo le debe mucho a las compañías de Little Theatre, debido a que estos grupos teatrales produjeron muchas de sus obras, divulgándolas por todo el mundo.

Además de Ibsen, los dramaturgos Björnstjerne Björnson y August Strinberg también influyeron notablemente sobre la dramaturgia moderna y sobre el movimiento del Little Theatre. Muchas de las obras de estos autores se produjeron en los escenarios de estas compañías de teatro experimental. Se trataban de dramas centrados en las emociones y en los problemas de la vida moderna.

No sólo estos tres dramaturgos escandinavos ejercieron un notable influjo sobre el pensamiento de los fundadores del movimiento teatral del Little Theatre en Europa, sino que además fue determinante la aparición de una serie de conceptos nuevos: un mayor deseo de libertad; el enfoque de los novelistas del Naturalismo; el conocimiento de la existencia de nuevas fuerzas en el pensamiento humano (los descubrimientos de la ciencia, las teorías de Darwin, el Determinismo, la psicología experimental, la aparición de una conciencia social, un mayor deseo de conocer la verdad, y la búsqueda científica de ésta) ${ }^{3}$. De todos estos conceptos, los novelistas naturalistas (especialmente Zola) y la teoría del Determinismo fueron los que mayor repercusión tuvieron sobre el pensamiento de los fundadores del movimiento teatral del Little Theatre.

En 1881 Zola publicó el ensayo "Le Naturalisme au Théâtre", basado en la aplicación de la teoría del Naturalismo a la literatura, tanto a la novela como al teatro. Este movimiento tenía como origen unos estudios publicados por el fisiólogo Claude Bernard, considerado el padre de la medicina experimental, que afirmaba que el uso de la experimentación en la investigación era de gran utilidad, no sólo para el estudio de la Física y la Química, sino además para el conocimiento de la

\footnotetext{
3 El Determinismo era una corriente filosófica que establecía que todo acontecimiento humano, incluyendo el pensamiento humano y las acciones, se encontraba determinado por una cadena de causa-consecuencia, según la cual, nada sucedía al azar.
} 
Fisiología, la Medicina y la observación del cuerpo humano. Zola tenía la intención de trasladar el Naturalismo a la literatura, con el objeto de llevar a cabo un estudio de la conciencia del hombre, pues consideraba que el método experimental ofrecería al investigador literario un conocimiento mayor sobre el intelecto y las emociones del Hombre. No obstante, Zola no especificaba el método para llevar a cabo este experimento, pues lo desconocía; sin embargo, sí sugirió que éste debía basarse en la imaginación creativa del artista y que esta actitud experimental sería muy positiva para el teatro:

I am attempting no justification of my own cause, [he writes] I am merely expressing my profound conviction--upon which I particularly insist--that the experimental and scientific spirit of the century will enter the domain of the drama, and that in it lies its only possible salvation4. (Miller, 1931: 15).

Cuatro años después de que Zola publicara el ensayo "Le Naturalisme au Théâtre", un joven admirador suyo, llamado André Antoine, fundó en París el primer Little Theatre en Europa, Théâtre Libre (1887). André Antoine era un trabajador de la Compañía de Gas de París, un hombre que sentía una gran pasión por el teatro, así como una persona dotada de un entusiasmo infinito y de un enorme poder de persuasión sobre los demás:

a man of the people, beginning his career as a clerk; a man imbued with a passionate yet clearsighted love of the stage; a man filled with an enthusiasm for the art of the theatre so great that he was able to enkindle all those with whom he came in contact, whether clerks and artisans who wanted to act, or men of great gifts who wanted to write (or were writing), plays -- men such as Eugene Brieux, Camille Fabre, Pierre Wolf, and George Ancey5. (MacKay, 1917:2).

\footnotetext{
4 Traducción de la cita: “No intento justificar mis razones -escribe- sino que simplemente expreso mi más profunda convicción (sobre la que insisto de manera especial), de que el espíritu científico y experimental del nuevo siglo penetrará en la dramaturgia y en éste se encuentra su única salvación".

5 Traducción de la cita: “Un hombre del pueblo, que comenzó trabajando de dependiente; un hombre con un amor apasionado, a la par que lúcido por el teatro; un hombre repleto de tal entusiasmo hacia el arte teatral que era capaz de contagiarlo a todos aquellos con los que hablaba, ya fueran vendedores o artesanos que querían actuar, hombres de gran talento que querían escribir (o
} 
Antoine era un genio en cuya persona se entremezclaban diferentes valores. Por un lado, en Antoine se aunaban el conocimiento instintivo, una gran empatía hacia los libros y las personas y un carácter muy independiente. Además, Antoine poseía amabilidad y equidad, así como frialdad y confianza en si mismo. En este sentido, Antoine jamás pedía consejo, él mismo seleccionaba todos los programas y no se dejaba influir por nadie, ni por amigos, ni tan siquiera por Zola, por quién sentía verdadera admiración. Por último, Antoine era un autócrata, sin embargo, el Théâtre Libre fue, en lo que respecta al número de dramaturgos, a la variedad de obras, así como al espíritu de esta compañía, el teatro más democrático de todos aquellos que pertenecieron al movimiento experimental europeo.

En cuanto a los ideales de Antoine, éstos se fundamentaban en los siguientes aspectos: por un lado, Antoine reconocía que tanto a los dramaturgos jóvenes como a los veteranos les resultaba muy difícil encontrar un teatro que les produjesen sus obras. Por otro lado, comprendía que los directores de los grandes teatros no podían comprometer su dinero, ni el del resto de socios, en proyectos que resultaran arriesgados para su empresa, así como sabía que los teatros subvencionados por el Estado no apoyaban este tipo de proyectos.

La trayectoria del Théâtre Libre se dividió en tres períodos principalmente: una primera fase en la que se inició la actividad de esta compañía teatral, que abarcó los dos programas de la primavera de 1887, así como los de la temporada siguiente. Una segunda fase, desde octubre de 1888 a junio de 1893, los años en los que esta compañía cosechó sus mayores éxitos. Y por último, una tercera fase, desde noviembre de 1893 hasta abril de 1896, en la que se produjo la decadencia y posterior clausura de la compañía.

El Théâtre Libre introdujo una serie de novedades en el mundo del teatro. Por un lado, era más pequeño que el teatro comercial, por lo que creaba una sensación de cercanía entre los actores y el público. Por otro lado, el Théâtre Libre se trataba de un estaban escribiendo) obras de teatro, tales como Eugene Brieux, Camille Fabre, Pierre Wolf, y George Ancey". 
teatro anti-comercial, en el que los beneficios de la taquilla no interesaban al productor, dado que se financiaba por medio del pago de la suscripción de los socios, y en el que se prescindía de la venta de entradas en la taquilla. En cierto modo, este método de financiación fortaleció la impresión de proximidad en el público, dado que cada uno de los socios del teatro era partícipe de una pequeña parcela de éste. Las obras que se producían en el Théâtre Libre se basaban en críticas sobre la sociedad y las obras representativas de la escuela de Scribe y Sardou, las cuales evitaban reflejar la realidad de la vida moderna. Asimismo, en el Théâtre-Libre se producían obras naturalistas, expresadas de modo naturalista, pues Antoine tenía un apetito insaciable por obras nuevas, vitalistas y que expresaran libertad. Por esto mismo, rechazaba las obras basadas en la tradición y el Romanticismo. En este teatro se buscó la simplicidad en los efectos escénicos, más que la decoración sobrecargada; todo ello desarrollado en un marco realista, cuidado hasta el más mínimo detalle. Se destacó la experimentación artística, dado que Antoine sentía obsesión por este tema y defendía el trabajo inédito de los dramaturgos jóvenes, pues consideraba que el verdadero sentido de este teatro radicaba en el descubrimiento de valores nuevos. André adoraba todo lo nuevo que tuviera talento, por lo que en el Théâtre Libre se presentaron una gran variedad de géneros dramáticos. En definitiva, Antoine pretendió con su teatro crear un laboratorio de experimentación en el que los actores representaran su papel con total libertad, así como de forma realista, huyendo de fórmulas estrictas y de los convencionalismos de los teatros grandes, que abusaban de los grandes discursos y los monólogos interminables. En relación con esto, Antoine implantó en su teatro una nueva técnica que consistía en que el actor no se dirigía a su público, sino que interpretaba su papel dándole la espalda. La compañía del Théâtre Libre estaba formada por jóvenes profesionales, que después de finalizar la jornada laboral, acudían al teatro a trabajar con energía y entusiasmo. El Théâtre Libre instauró un ideal de representación, así como de producción teatral que sirvió de modelo a muchos otros teatros.

La aparición de la compañía teatral experimental Théâtre Libre influyó en la aparición, casi al mismo tiempo que ésta, de muchas otras compañías en París que compartían sus mismos ideales. Varias de ellas las fundaron miembros que 
pertenecían al Théâtre Libre. Una de las compañías que apareció al mismo tiempo que el Théâtre Libre fue el Théâtre de l'Application, creada con el objetivo de que fuera un estudio teatral para los jóvenes actores del conservatorio de la Comédie Française. Otra compañía fue le Cercle des Écoliers (1887), inaugurada la misma tarde que el Théâtre Libre se estrenó y formada por alumnos del crítico literario Faguet. Más tarde, en 1890 el poeta Paul Fort, fundó la compañía Théâtre d'Art en oposición al realismo extremo. En 1893 el director teatral Aurélien Lugné-Pöe fundó le Maison de l'CEuvre ("la casa del arte”), otro Little Theatre, en el que se rechazaba el Naturalismo, se impulsaba el Simbolismo y en el que se produjeron numerosas obras extranjeras ${ }^{6}$. En 1897 Oscar Méténier, uno de los dramaturgos del Théâtre Libre, fundó Le Grand Guignol, una compañía especializada en obras de teatro de un solo acto. Le Grand Guignol realizó numerosas giras por Inglaterra y los Estados Unidos y sirvió de inspiración a otras compañías que posteriormente se establecieron en estos países. En 1906 Robert d'Humières fundó Théâtre des Arts y encomendó la dirección de esta compañía a Rodolphe Darzens, que fue otra de las personas que trabajó con Antoine en el Théâtre Libre.

Más tarde, en 1913 el crítico Jacques Copeau fundó la compañía Théâtre du Vieux Colombier, en la que se produjeron obras de todas las épocas hasta su cierre por la irrupción de la Primera Guerra Mundial. No obstante, una vez que terminó la guerra reanudó su actividad, llegando incluso a visitar el Garrick Theatre de Nueva York, donde trabajó durante dos temporadas para finalmente volver a París donde estuvo en funcionamiento hasta 1924. En este mismo año, el periodista Jacques Hébertot fundó dos Little Theatres, Théâtre de Champs-Elysées y Comédie des ChampsElysées. Las producciones del Théâtre de Champs-Elysées eran muy elaboradas, pues se trataba de un teatro bastante grande, y poseían menor radicalismo que las obras que

\footnotetext{
6 El Simbolismo era una escuela poética, y, en general, artística, aparecida en Francia a fines del siglo XIX, que eludía nombrar directamente los objetos y prefería sugerirlos o evocarlos. Los escritores simbolistas rechazaban las tendencias anteriores del siglo (el romanticismo, realismo y naturalismo) y proclamaban que la imaginación era el modo más auténtico de interpretar la realidad.
} 
se producían en el Comédie des Champs-Elysées, más pequeño y experimental que el anterior.

En 1919 se creó la Humble's Théâtre du Petit Monde, que se distinguió por llevar a cabo sus funciones en diferentes teatros y por ser el primer Little Theatre fundado en Europa y los Estados Unidos especializado en público infantil. Las obras que se producían pertenecían en su mayoría al escritor Alfred Machard. Esta compañía sirvió como precedente a otros muchos proyectos similares a éste.

En 1921 el actor Charles Dullin, que no estaba interesado por la obra puramente realista, fundó el Atelier, donde produjo numerosas obras experimentales francesas, italianas y españolas. En 1922 el dramaturgo Gaston Bato fundó Chimére, que produjo mayormente las obras de los doce dramaturgos que trabajaban para la misma. Este Little Theatre cerró al año siguiente debido a la falta de recursos económicos.

En 1923 el poeta Jacques Hébertot fundó el Studio. Este Little Theatre era bastante pequeño y las obras que se producían en el Studio muy experimentales.

Durante la temporada de 1929-1930 aparecieron dos Little Theatre en París de procedencia norteamericana. Una de estas compañías fue el Théâtre Anglo-Américain fundado por Thomas Van Dycke, que había liderado además la compañía Dramahouse Players. Este Little Theatre se creó para angloparlantes que residían en París y el repertorio eran básicamente obras inglesas o norteamericanas. El otro Little Theatre se llamaba Théâtre Femina y había sido fundado por Carol M. Sax, que había sido una figura central del Vagamond Theatre de Baltimore, así como del Romany Theatre de Lexington, en Kentucky.

Otro Little Theatre que se estableció por esa época fue la Petite Scène, dirigido por el escritor francés Xavier de Courville y formado por un grupo de artistas y personas adineradas. En este Little Theatre las obras que producían eran reestrenos de comedias y operetas de los siglos diecisiete y dieciocho. 
El Théâtre de Verdure of the Pré-Catelan fue otro Little Theatre. Situado en el parque parisino Bois de Bologne, poseía un auditorio al aire libre; durante sus 15 años de funcionamiento produjo dramaturgia poética y comedia ligera. El Théâtre de Verdure of the Pré-Catelan significó un precedente para las compañías teatrales que trabajaban al aire libre.

Otra compañía fue New Pigalle Theatre, fundada por el Barón Henri de Rothschild, financiero y dramaturgo conocido por el pseudónimo de André Pascal. Este Little Theatre poseía uno de los equipos de escenario más modernos.

Además de los Little Theatre establecidos en París, en muchos otros lugares de Francia surgieron estas compañías teatrales, siendo las más destacadas las que se nombran a continuación. La compañía situada en Orange, Théâtre Antique d'Orange, que era un festival de verano de ópera y dramaturgia. En la Bretaña francesa apareció Le Théâtre Populaire de Sainte Anne d'Auray, fundado por los abades Joseph Le Bayon y Louis Cadic. Se trataba de un auditorio que se pensaba en principio que fuera al aire libre, sin embargo, finalmente se optó por cubrirlo con un techo de paja dado que en la Bretaña el clima es muy húmedo, por lo que posteriormente se diseñó una construcción más fuerte. Esta compañía estaba formada por actores de todas las edades, a los cuales no se les pagaba salario alguno, debido a que las entradas tenían un precio muy bajo.

\section{BIBLIOGRAFÍA:}

Bentley, E. (1946): The Playwright as Thinker, a Study in Modern Drama, Nueva York: Reynal \& Hitchcock.

Brockett, O. G. y Findlay R. R. (1973): Century of Innovation: A History of European and American Theatre and Drama since 1870, Englewood Cliffs, Nueva Jork: PrenticeHall, Inc.

Cole, T. y Chinoy, H. K. (1963): Directors on Directing: A Sourcebook of the Modern Theatre, Indianapolis: Bobbs-Merrill. 
Cheney, S. (1925): The Art Theater: Its Character as Differentiated from the Commercial Theater; Its Ideals and Organization; and a Record of Certain European and American Examples, Nueva York: A. A. Knopf.

Chiari, J. (1958): The Flight from Naturalism, Londres: Camelot Press.

Davis, T. C. (1994): George Bernard Shaw and the Socialist Theatre, Westport (Connecticut): Praeger.

Dickinson, T. H. (1927): An Outline of Contemporary Drama, Boston: Houghton Mifflin.

Drain, R. (1995): Twentieth-Century Theatre, a Sourcebook, Nueva York: Routledge.

Eaton, W. P. Y Dean, A. (1926): Little Theatre Organization and Management, for Community, University and School: Including a History of the Amateur in Drama, Nueva York: Appleton and Company.

Everdell, W. R. (1998): The First Moderns: Profiles in the Origins of Twentieth-Century Thought, Chicago: University of Chicago Press.

Fischer-lichte, E. (2001): History of European Drama and Theatre, London: Routledge.

Frick, J. W. y Vallillo S. M. (1994): Theatrical Directors: A Biographical Directory, Westport, Connecticut: Greenwood Press.

Heller, O. (1912): Henrik Ibsen: Plays and Problems, Boston: Houghton Mifflin Company Publishers.

Isaacs, E. J. R. (1927): Essays on the Arts of the Theatre, Boston: Little, Brown, and Company.

Knapp, B. L. (1988): The Reign of the Theatrical Director: French Theatre, 1887-1924, Nueva York: The Whitston Publishing Company.

Kurtz, M. (1999): Jacques Copeau: Biography of a Theater, Carbondale (Illinois): Southern Illinois University Press.

Lewisohn, L. (1923): Modern Drama, an Essay in Interpretation, Nueva York: BW Heubsch.

Mackay, C. D'Arcy (1917): The Little Theatre in the United State, New York: Henry Holt.

Miller, A. I. (1931): The Independent Theatre in Europe, 1887 to the Present, Nueva York: Ray Long \& Richard R. Smith.

Roberts, V. M. (1962): On Stage a History of Theatre, Nueva York: Harper \& Row. 
Shepherd-Barr, K. (1997): Ibsen and Early Modernist Theatre, Westport (Connecticut): Greenwood Press.

Styan, J. L. (1981): Modern Drama in Theory and Practice: Volume 1, Realism and Naturalism. Cambridge: Cambridge University Press.

Waxman, S. M. (1926): Antoine and the Theatre-Libre. Cambridge (Massachusetts): Harvard University Press. 